\title{
CD3D is associated with immune checkpoints and predicts favorable clinical outcome in colon cancer
}

\author{
Yi Yang ${ }^{\ddagger}{ }^{1}$, Yiwen Zang ${ }^{\ddagger}{ }^{1}$, Chuang Zheng ${ }^{\ddagger}{ }^{1}$, Zhenyang $\mathrm{Li}^{1}$, Xiaodong Gu ${ }^{1}$, Minwei Zhou ${ }^{1}$, \\ Zihao Wang ${ }^{1}$, Jianbin Xiang*,1, Zongyou Chen**,1 \& Yiming Zhou***,1 \\ ${ }^{1}$ Department of General Surgery, Huashan Hospital, Shanghai 200040, China \\ *Author for correspondence: xjbzhw@163.com \\ **Author for correspondence: zongyouchen@163.com \\ ***Author for correspondence: iron_zhou@hotmail.com \\ ₹ Authors contributed equally
}

Aim: The T cell receptor-CD3 complex has shown great potential in tumor therapy. However, there is currently no research on CD3D in tumors. Materials \& methods: Correlation between CD3D expression and clinical parameters and immune checkpoints of of colon adenocarcinoma (COAD) were analyzed. Results: CD3D decreased with increasing clinical stage and microsatellite status of COAD. Functional enrichment analysis revealed that CD3D is related to immune activation and regulation. Coexpression analysis indicated that CD3D is correlated with immune checkpoint and immune-infiltrated cells. Patients with higher expression of CD3D showed better clinical outcome. Conclusion: The findings suggest that the participation of CD3D may serve as a prognostic marker of COAD and may act as a guide in the development of immunotherapy.

First draft submitted: 15 August 2019; Accepted for publication: 18 December 2019; Published online: 9 January 2020

Keywords: CD3D • COAD • immune • survival

Local recurrence, lymph node metastasis and disseminated metastasis are the main causes of death in colon cancer patients $[1,2]$. Therefore, it is important to explore the pathogenesis of colon cancer and find a suitable therapeutic target to improve prognosis.

In recent years, tumor immunity has become a breakthrough point in the diagnosis and treatment of tumors [3]. Tumor immunology puts forward the concept of the 'tumor microenvironment' and proposes that the immune state of the tumor microenvironment can accurately reflect the host's immune response to tumors [4]. Current research has shown that the immune score is an independent predictor that can assess the risk of colon cancer recurrence more effectively and accurately than traditional tumor-node-metastasis staging, lymphatic vessel infiltration, tumor differentiation and microsatellite instability (MSI) status [5]. The immune score has a relatively variable prognostic value. It has been suggested that the immune score of colon cancer can be applied to classify cancer and to improve individualized treatment strategies, especially chemotherapy [6].

Tumor-infiltrating lymphocytes are an important component of the tumor microenvironment. T cells in tumorinfiltrating lymphocytes play a crucial role in tumor immunity, and activation of $\mathrm{T}$ cells is a key step in initiating adaptive immune response. $T$ lymphocyte activation requires the extracellular attachment of the $T$ cell receptor (TCR) to antigen-presenting cells and subsequent formation of dynamic signaling complexes [7]. CD3 consists of a protein complex and is composed of distinct chains ( $\gamma$ chain encoded by CD3G, $\delta$ chain encoded by CD3D and $\epsilon$ chain encoded by CD3E). These chains could be combined with TCR and the $\zeta$-chain (encoded by CD3Z) to form the TCR-CD3 complex, which has vital functions in T cell antigen recognition and signal transduction. CD3 molecules include proteins encoded by CD3D, CD3E, CD3G and CD3Z. In addition to the role of signal transduction in T-cell activation, they all have their own unique functions. For instance, CD3G participates in the dynamic regulation of the TCR expression at the cell surface. CD3D is involved in thymocyte differentiation. $\mathrm{CD} 3 \mathrm{E}$ plays an essential role in correct T-cell development. CD3Z plays an important role in intrathymic T-cell

Future Medicine 
Table 1. Clinical features of colon adenocarcinoma patients.

Clinical features

Number

Age (years)

$-\leq 60$

159

$->60$

387

Gender

- Female

262

- Male

284

MSI status

- MSS

333

- MSI-H

97

- MSI-L

97

Clinical stage

$-1$

- II 219

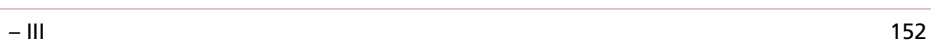

$\begin{array}{ll}- \text { IV } & 78\end{array}$

$\mathrm{N}$ stage

- NO 323

$-\mathrm{N} 1+\quad 223$

M stage

- MO 398

$\begin{array}{ll}-\mathrm{M} 1 & 78\end{array}$

Histology

- Colon adenocarcinoma 470

- Colon mucinous adenocarcinoma $\quad 71$

Neoadjuvant history

- No $\quad 542$

- Yes 4

Subdivision

$\begin{array}{ll}\text { - Cecum } & 127\end{array}$

- Ascending colon 104

- Descending colon $\quad 23$

- Transverse colon $\quad 45$

- Sigmoid colon $\quad 185$

- Hepatic flexure $\quad 33$

- Splenic flexure 9

- Rectosigmoid junction 1

MSI: Microsatellite instability; MSI-H: MSI high status; MSL-L: MSI low status; MSS: Microsatellite-stable.

differentiation. Studies have shown that CD3E can be used as an independent prognostic factor of head and neck tumor recurrence [8]. In lung cancer, a decrease in CD3E expression may lead to dysregulation of T-cell function [9]. However, there is currently no research on CD3D in tumors.

In this study, we evaluated the expression of CD3D in various tumors, including colon adenocarcinoma (COAD). We then examined the correlation between CD3D, clinical phenotypes of COAD and MSI status. Functional enrichment and protein-protein interaction (PPI) network analysis of CD3D-related genes revealed that CD3D is strongly linked to immune activation and regulation. In addition, we evaluated the correlation between CD3D and immune score and checkpoint. We further investigated the relationship between CD3D and lymphocytes, immunomodulators and chemokines. Ultimately, we demonstrated that low expression of CD3D is an index of poor prognosis in colon cancer patients. 


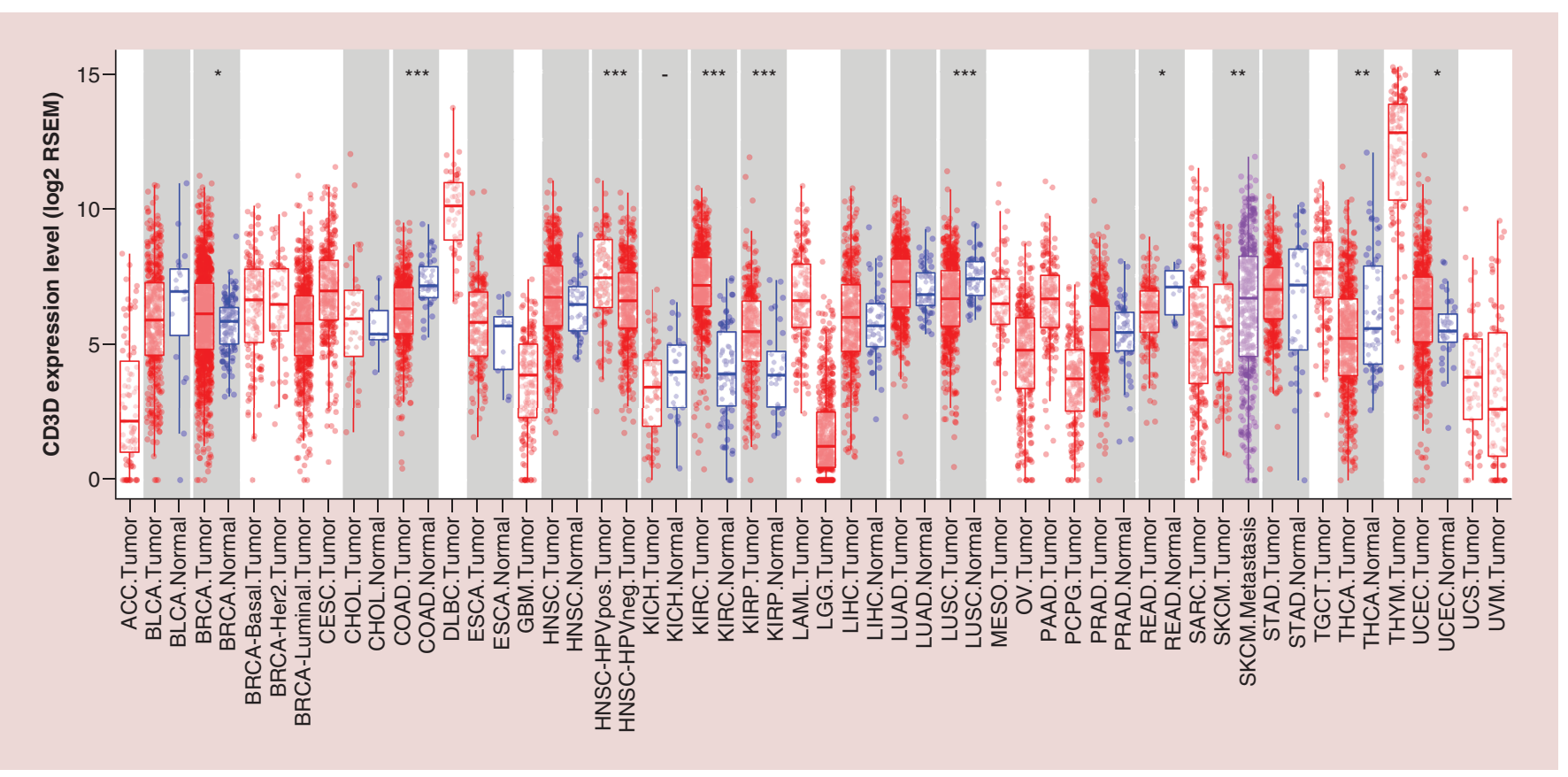

Figure 1. Relative expression of CD3D in various carcinomas. Red, blue and purple dots represent tumor tissues, normal tissues and metastatic tissues, respectively. * ** and $* * *$ indicate $p<0.05,0.01$ and 0.001 , respectively.

ACC: Adrenocortical carcinoma; BLCA: Bladder urothelial carcinoma; BRCA: Breast invasive carcinoma; CESC: Cervical squamous cell carcinoma and endocervical adenocarcinoma; CHOL: Cholangiocarcinoma; COAD: Colon adenocarcinoma; DLBC: Lymphoid neoplasm diffuse large B-cell lymphoma; ESCA: Esophageal carcinoma; GBM: Glioblastoma multiforme; HNSC: Head and neck squamous cell carcinoma; KICH: Kidney chromophobe; KIRC: Kidney renal clear cell carcinoma; KIRP: Kidney renal papillary cell carcinoma; LAML: Acute myeloid leukemia; LGG: Brain lower grade glioma; LIHC: Liver hepatocellular carcinoma; LUAD: Lung adenocarcinoma; LUSC: Lung squamous cell carcinoma; MESO: Mesothelioma; OV: Ovarian serous cystadenocarcinoma; PAAD: Pancreatic adenocarcinoma; PCPG: Pheochromocytoma and paraganglioma; PRAD: Prostate adenocarcinoma; READ: Rectum adenocarcinoma; SARC: Sarcoma; SKCM: Skin cutaneous melanoma; STAD: Stomach adenocarcinoma; TGCT: Testicular germ cell tumors; THCA: Thyroid carcinoma; THYM: Thymoma; UCEC: Uterine corpus endometrial carcinoma; UCS: Uterine carcinosarcoma; UVM: Uveal melanoma.

Based on the TCGA databank, this study is the first to investigate the correlation between CD3D and COAD and indicates the potential importance of $\mathrm{CD} 3 \mathrm{D}$, which could be an important finding with regard to the future of tumor immunotherapy.

\section{Materials \& methods}

\section{Individuals \& clinical information}

RNA-seq data and clinical information were acquired from the Genomic Data Commons portal, an official data portal of TCGA [10]. Samples were collected from primary tumor (Table 1) and expression of genes was normalized by the edgeR package [11]. To avoid noise, we only selected mRNAs with normalized expression equal to or above 1 in at least $10 \%$ of the samples for downstream analysis. Overall survival was calculated from the date of diagnosis to the date of death or last follow-up.

\section{Immune correlation analysis}

To investigate the function of CD3D in colon cancer, Pearson coefficient correlation analysis was performed to identify CD3D-related genes with a cutoff of $|r|>=0.4$ and $p<0.5$. The clusterProfiler R package was applied to investigate gene ontology terms of CD3D-related genes related to biological process (BP), molecular function and cellular component [12].

The STRING website was utilized to analyze the PPI network of CD3D-related genes [13]. The Cytoscape app was employed to re-analyze the PPI network based on topology using the MCODE plug-in to identify the most connected networks [14].

The immune score of each sample was calculated using the ESTIMATE R package [15]. The immune score of the colon cancer samples was classified as either high (higher than median) or low (lower than median). 

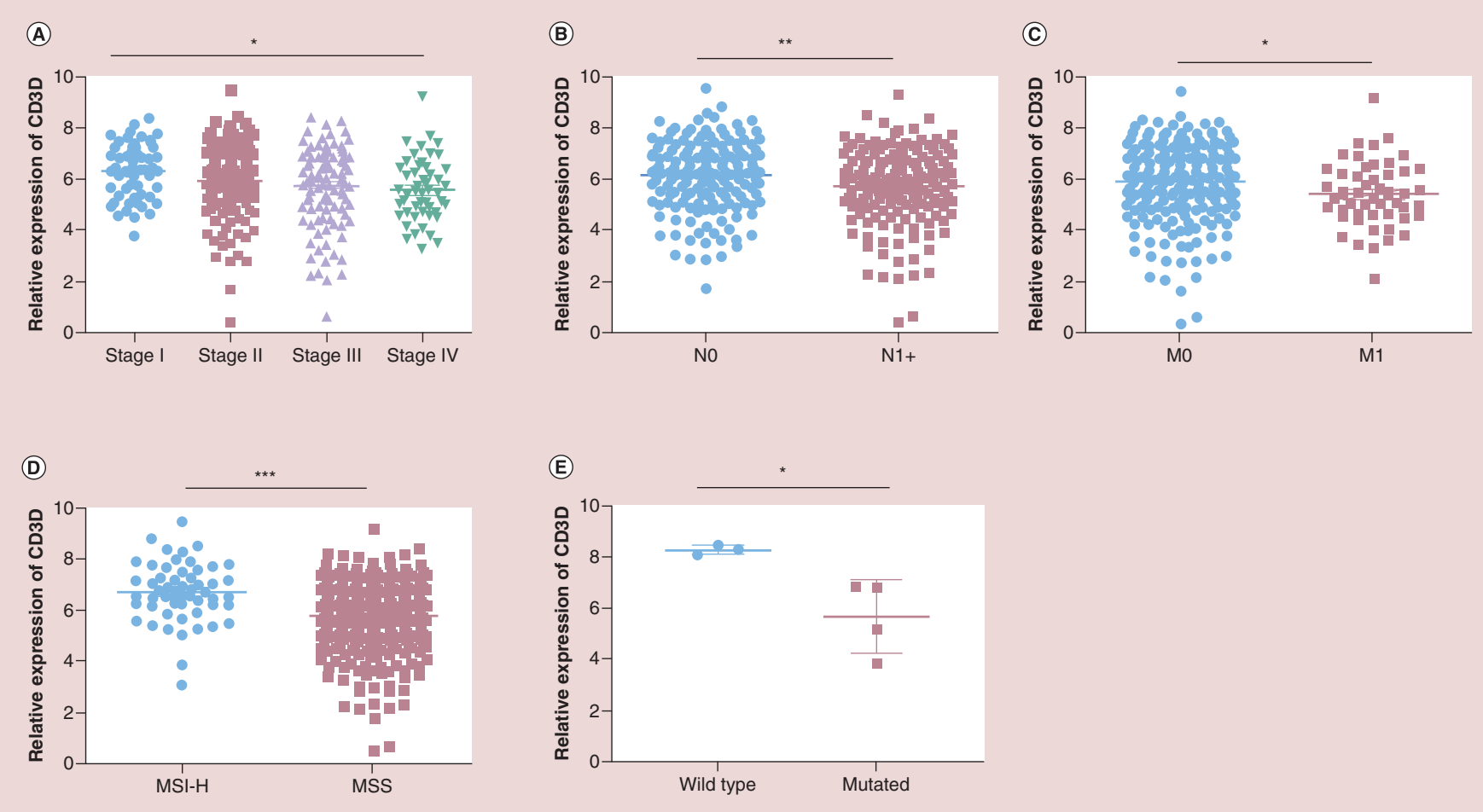

Figure 2. Relative expression of CD3D in subgroups of colon adenocarcinoma. CD3D was downregulated (A) with increasing clinical stage, (B) with lymph node metastasis, (C) with distance metastasis, (D) in the MSS subgroup and (E) in the Kras status subgroup. * ** and $* * *$ indicate $p<0.05,0.01$ and 0.001 , respectively. MSS: Microsatellite-stable.

The tumor-immune system interactions database (TISIDB) website was adopted to investigate the relevance between CD3D and lymphocytes, major histocompatibility complex molecules and chemokines [16].

\section{Statistical analysis}

The difference between two groups was analyzed by unpaired student's $t$ test and that between three or more groups was subjected to analysis of variance. In survival analysis, patients were classified as high or low expression group according to the optimal cut-off point calculated by the Contal and O'Quigley method. Survival analysis was conducted using Kaplan54-Meier curves with log-rank test.

\section{Results}

\section{CD3D was downregulated in COAD}

To explore the role of CD3D in tumors, we first investigated the relative expression of CD3D between tumors and corresponding normal tissues using the TIMER website [17]. We found that CD3D was significantly downregulated in various carcinomas, including colorectal cancer, lung squamous cell carcinoma and thyroid carcinoma. However, CD3D was upregulated in breast cancer and kidney renal clear cell carcinoma (Figure 1).

\section{CD3D was correlated with clinical stage \& microsatellite status in COAD}

The expression of CD3D was investigated with regard to clinical stage. The difference between two groups was analyzed by unpaired student's $t$ test and the difference between three or more groups was subjected to analysis of variance. Results revealed that $\mathrm{CD} 3 \mathrm{D}$ was significantly suppressed in the late stage compared with the early stage (Figure 2A). In addition, CD3D was decreased in patients with lymph node metastasis (Figure 2B) and distance metastasis (Figure 2C). Microsatellite status, which is a fundamental factor in predicting the clinical outcome of COAD and chemotherapeutic response, showed a dramatic difference between COAD cases. Regarding the remarkable molecular heterogeneity of COAD, we further evaluated the expression of CD3D in COAD with different microsatellite status. Patients with microsatellite-stable tumors showed lower CD3D expression compared 


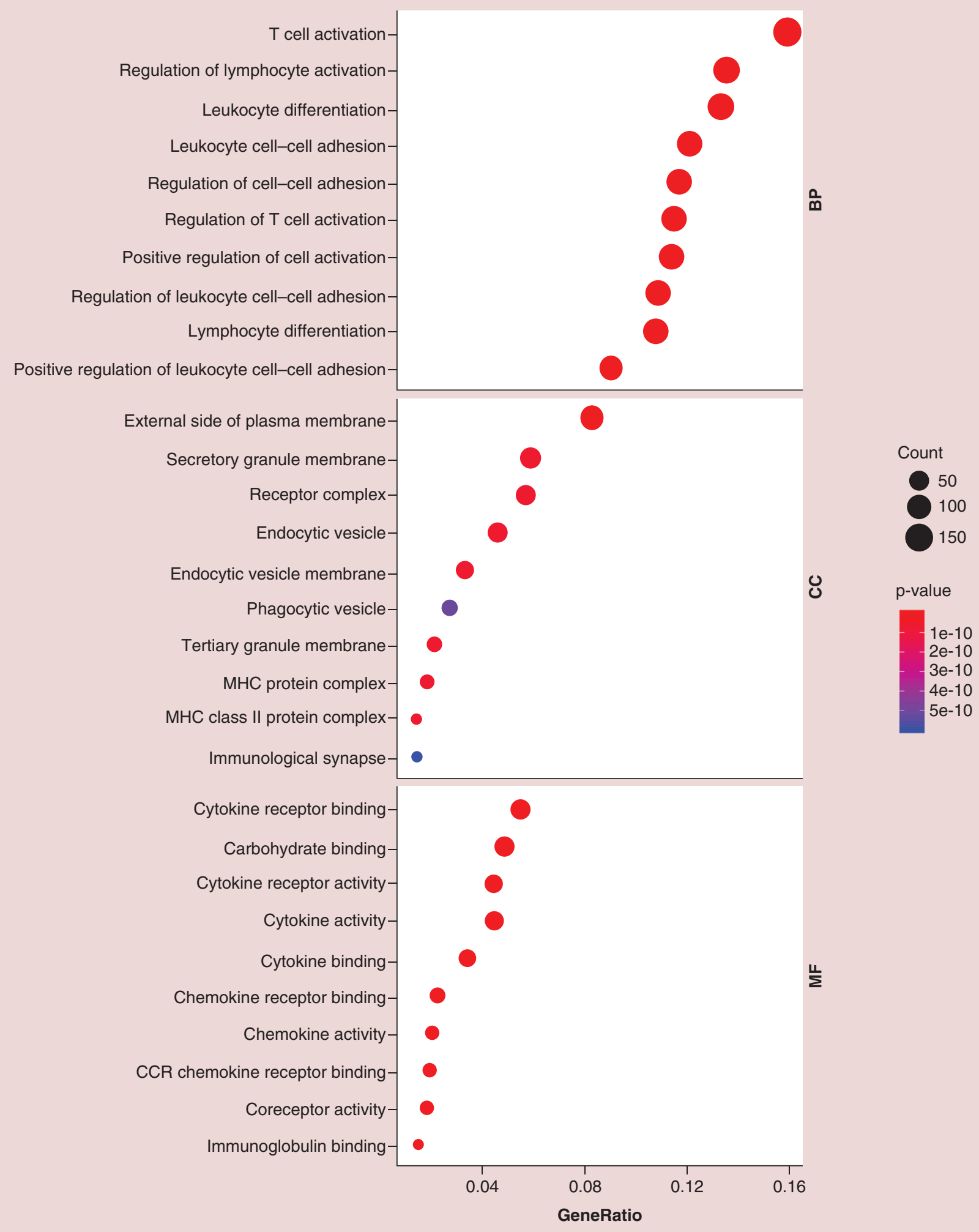

Figure 3. Gene ontology analysis of CD3D-related genes in biological process, cellular component and molecular function categories. The size of each dot indicates the number of genes in this term and the color of each dot indicates the p-value of this term. 


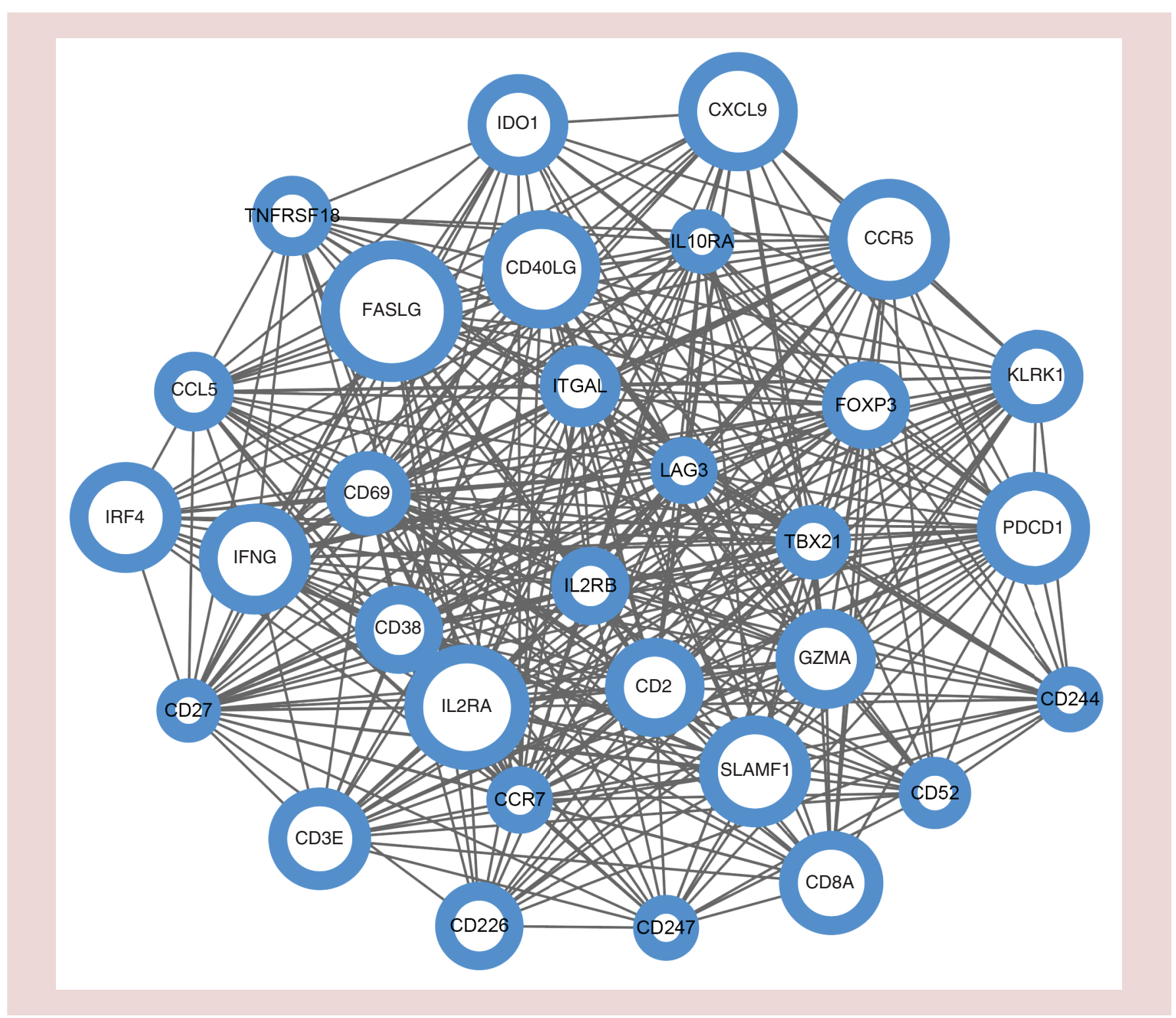

Figure 4. Protein-protein interaction network analysis of CD3D-related genes. The cluster was analyzed by STRING database and reanalyzed by the MCODE plugin. The size of each dot indicates the MCODE score calculated by MCODE and the segment between two nodes represents the interaction between these two genes.

with those with high (MSI-H) status (Figure 2D). These findings suggested that CD3D is closely correlated with clinical stage and microsatellite status in COAD. In addition, we tried to figure out the relation between CD3D with Kras or Braf mutation. As shown in Figure 2E, the expression of CD3D is higher in the wild type of Kras. Unfortunately, according to the status of Braf, there is only one sample with mutated Braf and six samples with wild type Braf, thus the correlation between CD3D and the Braf status was not analyzed.

\section{CD3D participated in immune-related processes in COAD}

To explore the function of CD3D in COAD, we identified 1100 CD3D-related genes using Pearson coefficient analysis. Gene ontology analysis performed by clusterProfiler R package revealed enrichments in molecular function, $\mathrm{BP}$ and cellular component terms, suggesting that the CD3D-related genes were mainly involved in the regulation of immunocytes, cytokines (especially of $\mathrm{T}$ cells and leukocytes), and major histocompatibility complex protein complexes, respectively (Figure 3). 


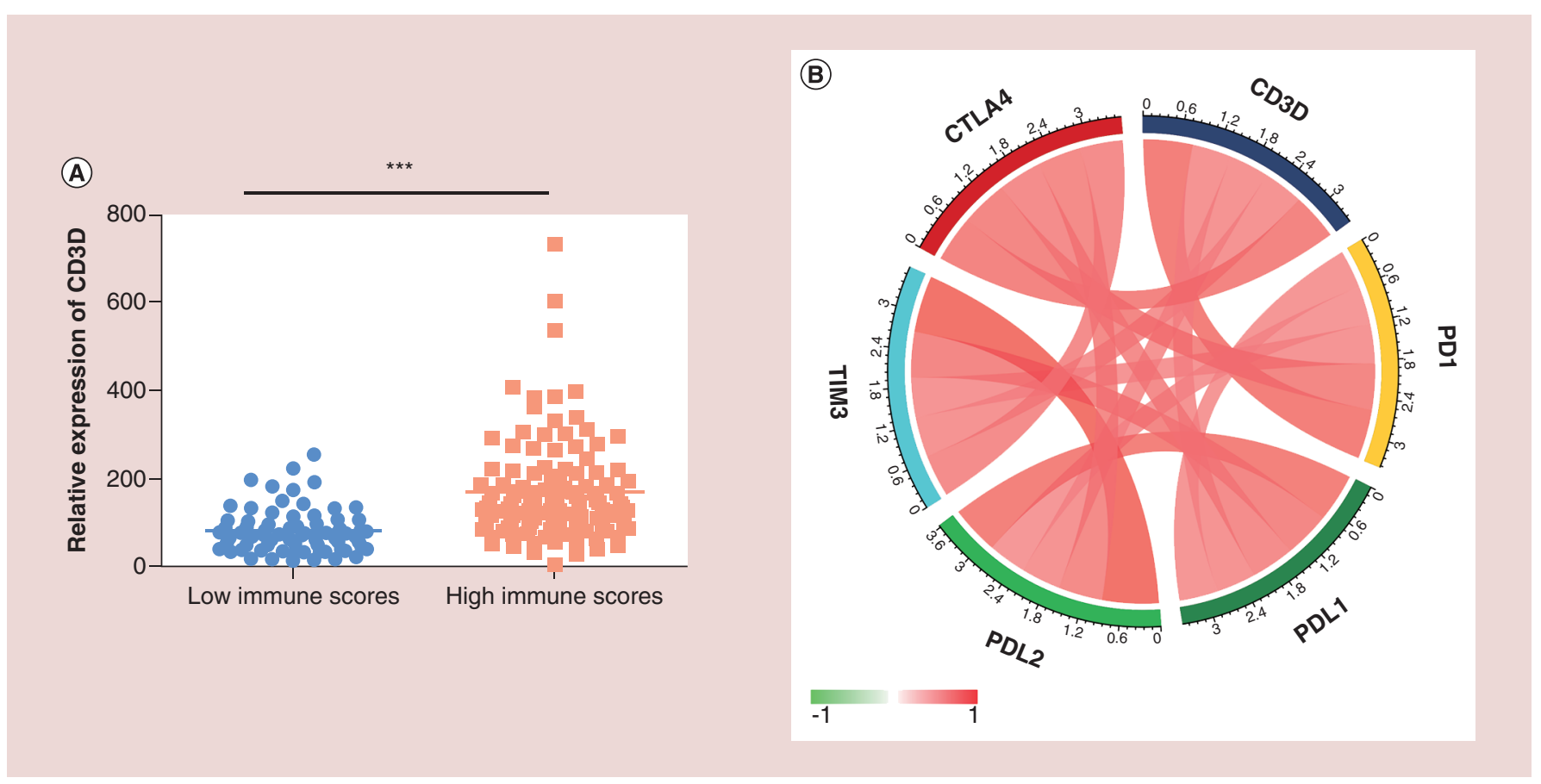

Figure 5. CD3D was highly correlated with immune score and immune checkpoint. (A) CD3D was enriched in samples with high immune scores compared with that in samples with low immune scores. (B) CD3D was highly correlated with immune checkpoints of COAD. The color shade of each segment indicates the correlation between two genes, where red and green represent positive and negative correlation, respectively. $* * *$ indicates $\mathrm{p}<0.05$.

COAD: Colon adenocarcinoma.

PPI network analysis constructed by STRING database was carried out based on the CD3D-related genes with correlation of greater than 0.7. This network, which contained 177 nodes and 2096 interactions, was too complicated and did not provide effective information. Therefore, the MCODE plugin of the Cytoscape software was utilized to disintegrate them given the topology of the entire network. The cluster with the highest score was selected, which contained 31 nodes and 359 edges. Among these nodes, Fas Ligand (FASLG), which had the highest node score, was considered as the seed gene. Others like Interleukin 2 Receptor Subunit Alpha (IL2RA), C-C Motif Chemokine Receptor 5 (CCR5), C-X-C Motif Chemokine Ligand 9 (CXCL9), CD40 Ligand (CD40LG), Programmed Cell Death 1 (PDCD1), Signaling Lymphocytic Activation Molecule Family Member 1 (SLAMF1), Interferon Regulatory Factor 4 (IRF4) and IFN- $\gamma$ also showed tight linkage with other genes (Figure 4).

The results mentioned above suggested that CD3D may be involved in the immune regulation network of colon cancer. Thus, we evaluated the immune scores of COAD samples based on the ESTIMATE algorithm. Samples were divided into the low and high immune score groups, and the difference in CD3D expression between the two groups was calculated. As shown in Figure 5A, samples with high immune scores showed an increase in CD3D expression compared with those low immune scores.

\section{CD3D was associated with immune checkpoints in COAD}

As shown above, the functions of CD3D-related genes were mainly enriched in immune-related BPs in COAD. Therefore, the correlation between $\mathrm{CD} 3 \mathrm{D}$ and immune checkpoints, such as programmed cell death protein 1 (PD1), programmed cell death 1 ligand 1 (PDL1), programmed cell death 1 ligand 2 (PDL2), $\mathrm{T}$ cell immunoglobulin mucin 3 (TIM3) and cytotoxic T-lymphocyte-associated protein 4 (CTLA4), was evaluated by Pearson correlation analysis (Figure 5B). Surprisingly, the correlation coefficient was 0.794 between CD3D and PD1 and 0.751 between CD3D and CTLA4. 


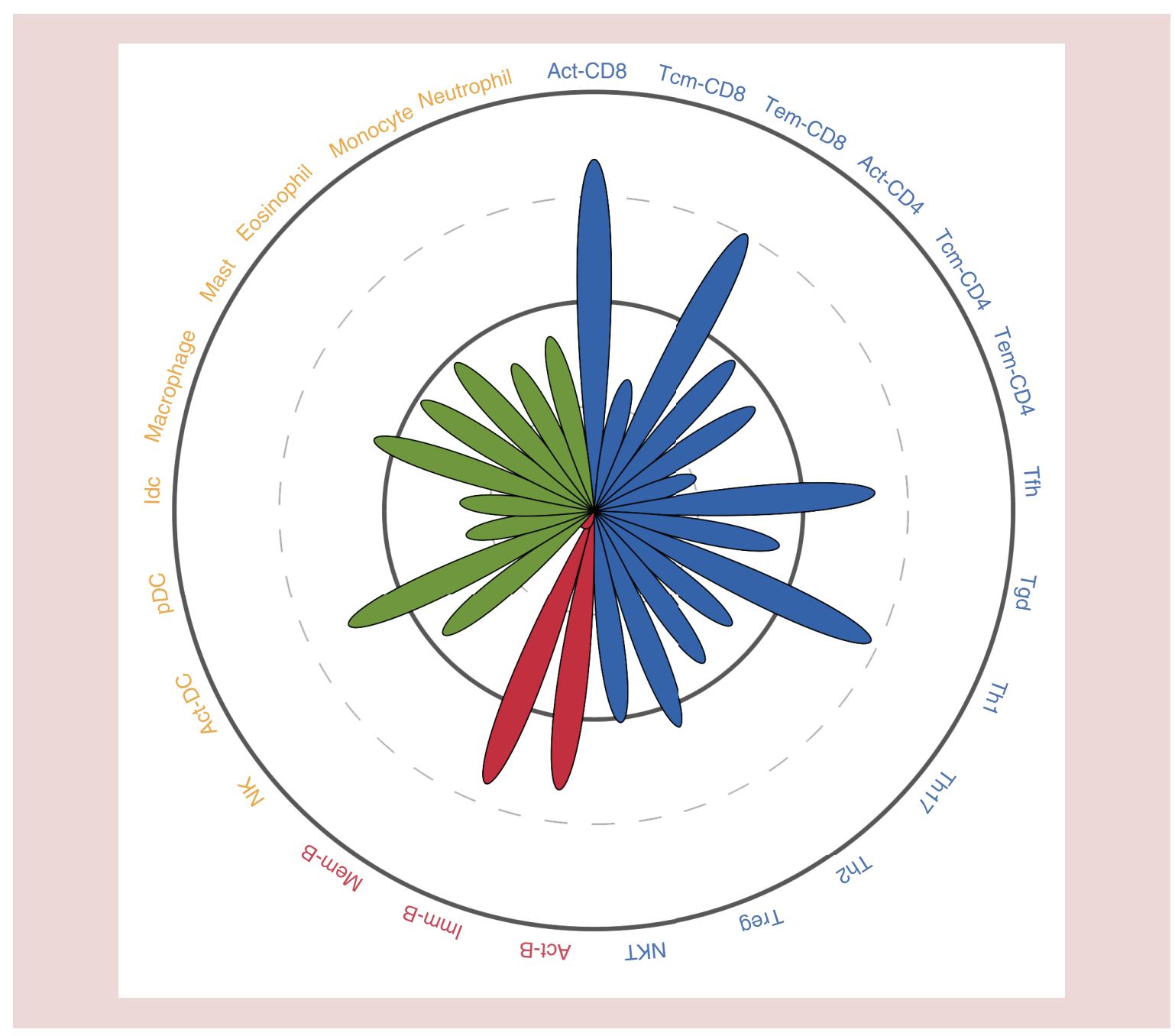

Figure 6. The correlation between CD3D and lymphocytes. Blue, red and yellow represent T cells, B cells and other lymphocytes, respectively. The length of each leaf represents the correlation between CD3D and lymphocytes. The correlation of the four circles are $0.25,0.5,0.74$ and 1 , respectively.

CD3D was strongly related with T lymphocytes in COAD

Using the TISIDB website, we first explored the association between CD3D and lymphocytes and showed that $\mathrm{CD} 3 \mathrm{D}$ has a strong correlation with activated $\mathrm{CD}^{+} \mathrm{T}$ cells, effector memory $\mathrm{CD} 8^{+} \mathrm{T}$ cells and $\mathrm{Th} 1$ cells (Figure 6).

\section{CD3D predicted favorable survival in COAD}

Given the strong relationship between $\mathrm{CD} 3 \mathrm{D}$ and the immune status of $\mathrm{COAD}$, we explored the prognostic value of CD3D in COAD using Kaplan-Meier curves with log-rank test. Individuals with higher CD3D expression demonstrated favorable clinical outcome compared with those with lower CD3D expression (Figure 7A). Considering the diverse therapeutic strategies based on microsatellite status, we further analyzed the predictive effect of $\mathrm{CD} 3 \mathrm{D}$ in subgroups of microsatellite status in COAD. In the microsatellite-stable subgroup, patients with increasing expression of $\mathrm{CD} 3 \mathrm{D}$ showed promising prognosis compared with those low $\mathrm{CD} 3 \mathrm{D}$ expression (Figure $7 \mathrm{~B}$ ). As there were a limited number of patients in the MSI-H group, we did not investigate the prognostic value of CD3D in this subgroup. Additionally, the prognostic value of CD3D was evaluated by clinical stage. In stages I, III and IV (Figure $7 \mathrm{C}, \mathrm{E}$ and $\mathrm{F}$, respectively), $\mathrm{CD} 3 \mathrm{D}$ served as a promising prognostic index, but we did not observe a significance difference at stage II of COAD (Figure 7D). 
(A)
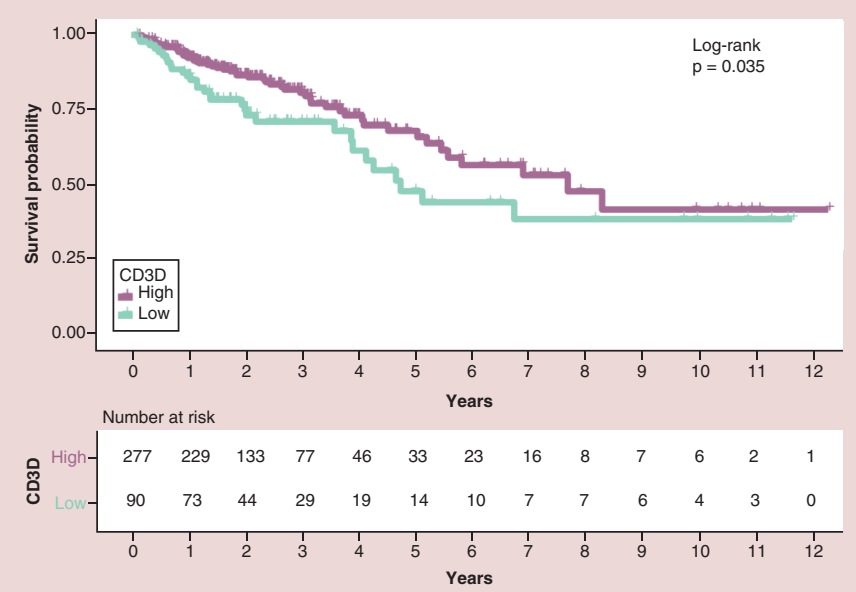

(C)
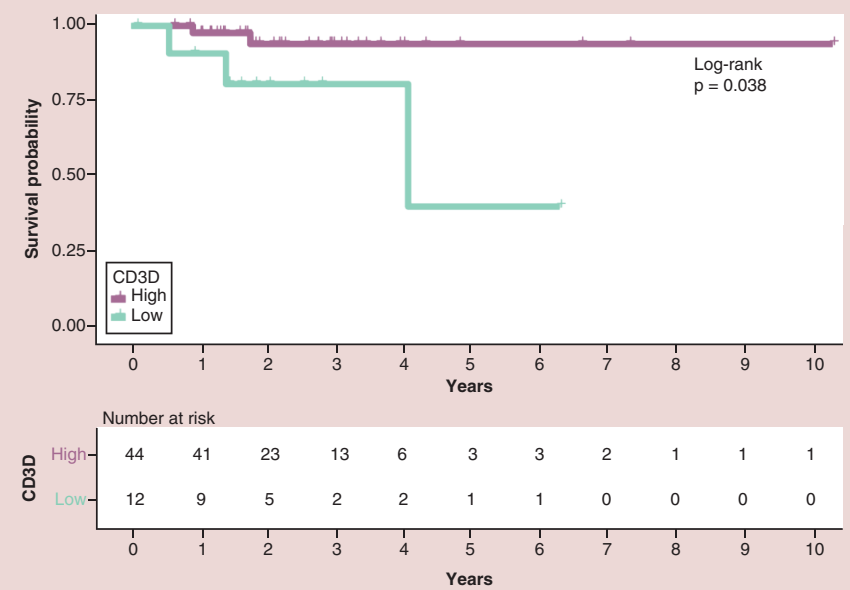

(E)

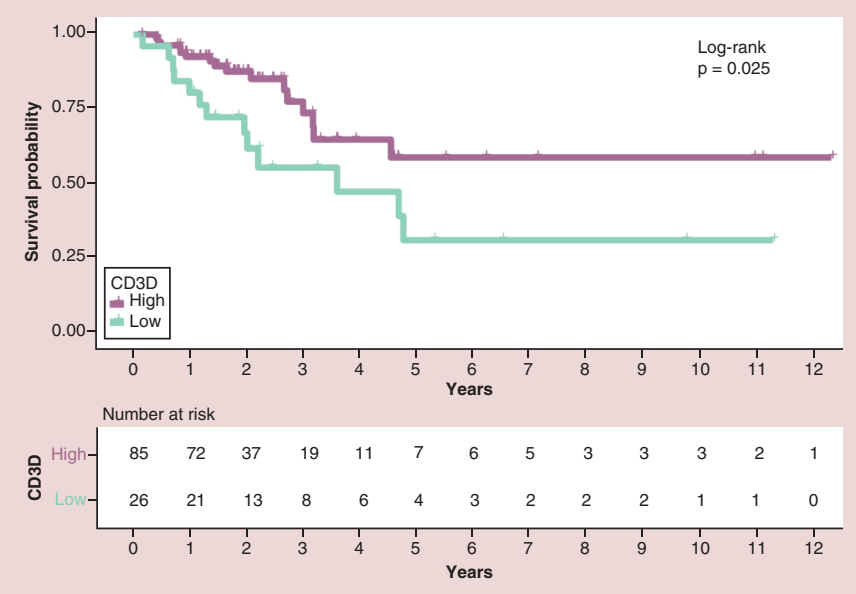

(B)
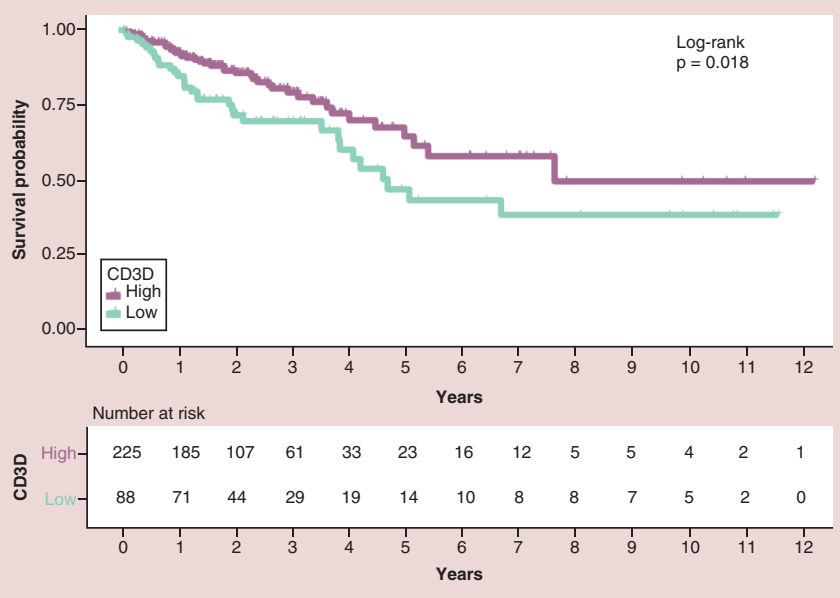

(D)

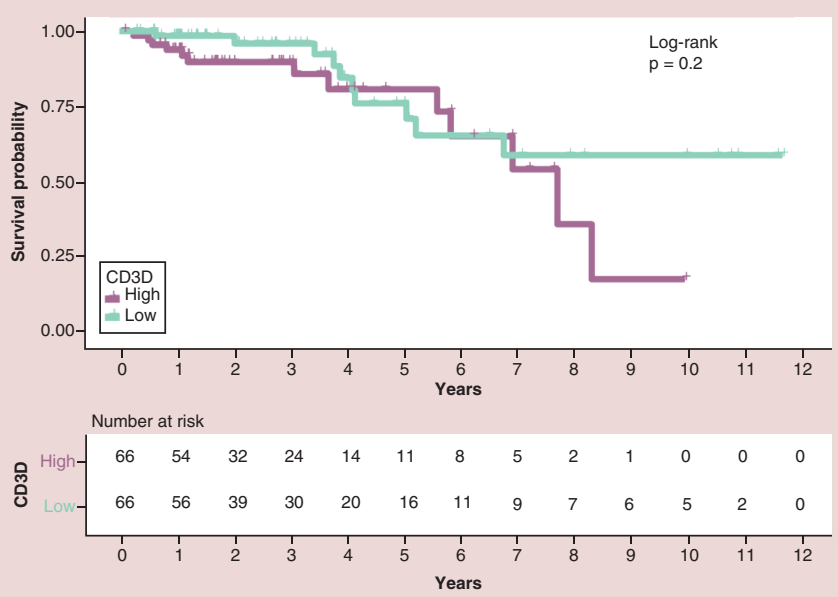

(F)

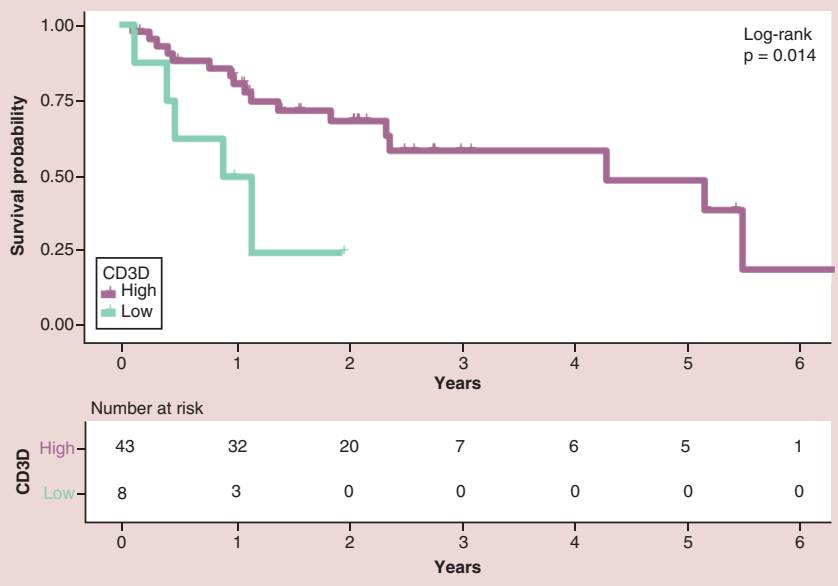

Figure 7. Survival analysis of CD3D in colon adenocarcinoma. Detection of the correlation between CD3D and (A) clinical outcome, (B) microsatellite status and clinical stage (C) I, (D) II, (E) III and (F) IV. High expression of CD3D predicts favorable clinical outcome in COAD in the MSS group and clinical stage I, III and IV. No significant difference was observed between high and low expression of CD3D at stage II. COAD: Colon adenocarcinoma; MSS: Microsatellite-stable. 


\section{Discussion}

Even if comprehensive therapeutic strategies have been designed to treat COAD, the clinical outcome is still unsatisfactory. With the advancement of immunotherapy, increasing attention has been devoted to the homogeneous immune status of COAD.

Malignant tumors were previously thought to consist of homogeneous and uniformly distributed tumor cells. However, modern oncology proposes that malignant tumors are not only composed of a single tumor cell type, but a variety of complex tissues of different cell types also contribute to tumor progression. This is known as the tumor microenvironment and is made up of the extracellular matrix around the tumor. It contains not only tumor cells, but also stromal cells, inflammatory cells, vascular components and extracellular matrix proteins [18]. The tumor microenvironment plays an important role in the occurrence and development of malignant tumors. For example, it can inhibit the activation of effector lymphocytes, reduce their infiltration ability and inhibit the function of effector lymphocytes that have infiltrated the tumor tissue. Moreover, the tumor microenvironment can cause insufficient draining of the surface antigens of tumor cells by lymph nodes, reduce the sensitization of effector lymphocytes and impair or delete the normal antitumor immunity of the body.

In this study, we discovered that CD3D was significantly decreased in COAD. The expression of CD3D was downregulated with increasing clinical stage, and a close relationship was observed between CD3D and microsatellite status. Functional enrichment analysis revealed that CD3D is highly related to immune activation and regulation in $\mathrm{COAD}$, suggesting the participation of $\mathrm{CD} 3 \mathrm{D}$ in the immune response of COAD. Coexpression analysis revealed that $\mathrm{CD} 3 \mathrm{D}$ is strongly correlated with immune score, immune checkpoint and immune-infiltrated cells. Furthermore, patients with higher expression of CD3D showed better clinical outcome compared with those lower CD3D expression. The observations collectively demonstrated that CD3D may serve as a prognostic marker of COAD and may guide the development of immunotherapy.

Immunotherapy, an important field in cancer treatment research, has become the most promising way to cure tumors after surgery, radiotherapy, chemotherapy and molecular targeted therapy [19]. Tumor immunotherapy is carried out by stimulating and enhancing the immune function of the body to control and kill tumor cells. T lymphocytes are the core of the body's antitumor immune response. According to the difference in T-cell surface cluster of differentiation antigen molecules, $\mathrm{T}$ lymphocytes can be divided into $\mathrm{CD} 4^{+}$and $\mathrm{CD} 8{ }^{+} \mathrm{T}$ cells. $\mathrm{CD} 4^{+} \mathrm{T}$ cells are mainly helper $\mathrm{T}$ cells, whereas $\mathrm{CD} 8^{+} \mathrm{T}$ cells are mainly cytotoxic $\mathrm{T}$ cells that can kill tumor cells through cytotoxicity. $\mathrm{CD}^{+} \mathrm{T}$ cells are activated and become Th cells, which are divided into Th1 and Th2 subtypes. Th1 cells affect cellular immunity and assist in the initiation of cytotoxic T lymphocytes, with critical functions in antitumor immunity. Here, we found that there was a strong correlation between CD3D and infiltrating immune cells, especially $\mathrm{CD}^{+} \mathrm{T}$ cells and Th1 cells, suggesting that CD3D could serve as a biomarker of the degree of $\mathrm{CD}^{+} \mathrm{T}$ cell and Th1 cell infiltration. Along with the strong correlation between CD3D and immune checkpoint, $\mathrm{CD} 3 \mathrm{D}$ may be a potential therapeutic target in immune therapy.

\section{Conclusion}

In brief, $\mathrm{CD} 3 \mathrm{D}$ was decreased with increasing clinical stage of COAD. Biological functional analysis revealed that $\mathrm{CD} 3 \mathrm{D}$ principally participated in immune activation and regulation and was highly correlated with immune checkpoint and $\mathrm{T}$ lymphocytes, especially $\mathrm{CD}^{+} \mathrm{T}$ cells. Survival analysis revealed $\mathrm{CD} 3 \mathrm{D}$ as a favorable index in the clinical outcome of COAD. Taken together, CD3D may be a therapeutic target in future immune therapy. Further experimental research is required to verify the results of our research.

\section{Future perspective}

COAD is an immunogenic tumor. The TCR-CD3 complex has vital functions in T-cell antigen recognition and signal transduction, which may be the answer for immunotherapy of COAD.

- Coexpression analysis indicated that CD3D is strongly correlated with immune score, immune checkpoint and immune-infiltrated cells.

- Patients with higher expression of CD3D showed better clinical outcome. 
Financial \& competing interests disclosure

The authors have no relevant affiliations or financial involvement with any organization or entity with a financial interest in or financial conflict with the subject matter or materials discussed in the manuscript. This includes employment, consultancies, honoraria, stock ownership or options, expert testimony, grants or patents received or pending, or royalties.

\section{Ethical conduct of research}

The authors state that they have obtained appropriate institutional review board approval or have followed the principles outlined in the Declaration of Helsinki for all human or animal experimental investigations.

\section{Open access}

This work is licensed under the Attribution-NonCommercial-NoDerivatives 4.0 Unported License. To view a copy of this license, visit http://creativecommons.org/licenses/by-nc-nd/4.0/

\section{References}

1. Siegel RL, Miller KD, Jemal A. Cancer statistics, 2019. CA Cancer J. Clin. 69(1), 7-34 (2019).

2. Riihimäki M, Hemminki A, Sundquist J, Hemminki K. Patterns of metastasis in colon and rectal cancer. Sci. Rep. 6, 29765 (2016).

3. Raval RR, Sharabi AB, Walker AJ, Drake CG, Sharma P. Tumor immunology and cancer immunotherapy: summary of the 2013 SITC primer. J. Immunother. Cancer 14(2), 14 (2014).

4. Balkwill FR, Capasso M, Hagemann T. The tumor microenvironment at a glance. J. Cell Sci. 125(23), 5591-5596 (2012).

5. Galon J, Pagès F, Marincola FM et al. Cancer classification using the immunoscore: a worldwide task force. J. Transl. Med. 3(10), 205 (2012).

6. Roelands J, Kuppen PJK, Vermeulen L et al. Immunogenomic classification of colorectal cancer and therapeutic implications. Int. J. Mol. Sci. 18(10), (2017). doi:10.3390/ijms18102229

7. de la Hera A, Müller U, Olsson C, Isaaz S, Tunnacliffe A. Structure of the T cell antigen receptor (TCR): two CD3 epsilon subunits in a functional TCR/CD3 complex. J. Exp. Med. 173(1), 7-17 (1991).

8. Upreti D, Zhang ML, Bykova E, Kung SK, Pathak KA. Change in CD3̧-chain expression is an independent predictor of disease status in head and neck cancer patients. Int. J. Cancer 139(1), 122-129 (2016).

9. Prado-Garcia H, Aguilar-Cazares D, Meneses-Flores M, Morales-Fuentes J, Lopez-Gonzalez JS. Lung carcinomas do not induce T-cell apoptosis via the Fas/Fas ligand pathway but down-regulate CD3 epsilon expression. Cancer Immunol. Immunother. 57(3), 325-336 (2008).

10. Tomczak K, Czerwińska P, Wiznerowicz M. The Cancer Genome Atlas (TCGA): an immeasurable source of knowledge. Contemp. Oncol (Pozn). 19(1A), A68-A77 (2015).

11. Robinson MD, McCarthy DJ, Smyth GK. edgeR: a Bioconductor package for differential expression analysis of digital gene expression data. Bioinformatics 26(1), 139-140 (2010).

12. Yu G, Wang LG, Han Y, He QY. clusterProfiler: an R package for comparing biological themes among gene clusters. OMICS 16(5), 284-287 (2012).

13. von Mering C, Huynen M, Jaeggi D, Schmidt S, Bork P, Snel B. STRING: a database of predicted functional associations between proteins. Nucleic Acids Res. 31(1), 258-261 (2003).

14. Shannon P, Markiel A, Ozier O et al. Cytoscape: a software environment for integrated models of biomolecular interaction networks. Genome Res. 13(11), 2498-2504 (2003).

15. Yoshihara K, Shahmoradgoli M, Martínez E et al. Inferring tumour purity and stromal and immune cell admixture from expression data. Nat. Commun. 4, 2612 (2013).

16. Ru B, Wong CN, Tong $\mathrm{Y}$ et al. TISIDB: an integrated repository portal for tumor-immune system interactions. Bioinformatics 35, 4200-4202 pii: btz210 (2019).

17. Li T, Fan J, Wang B et al. TIMER: a web server for comprehensive analysis of tumor-infiltrating immune cells. Cancer Res. 77(21), e108-e110 (2017).

18. Quail DF, Joyce JA. Microenvironmental regulation of tumor progression and metastasis. Nat. Med. 19(11), 1423-1437 (2013).

19. Farkona S, Diamandis EP, Blasutig IM. Cancer immunotherapy: the beginning of the end of cancer? BMC Med. 14, 73 (2016). 